\title{
PROTOCOLO PARA EL MANEJO INICIAL DEL PACIENTE QUEMADO HOSPITAL UNIVERSITARIO DR. ALFREDO VAN GRIEKEN CORO -VENEZUELA, NOVIEMBRE - DICIEMBRE 2015
}

\author{
INITIAL MANAGEMENT PROTOCOL FOR PATIENT BURNED \\ UNIVERSITY HOSPITAL DR. ALFREDO VAN Grieken \\ CORO -VENEZUELA, NOVEMBER - DECEMBER 2015
}

Germánico Fuentes-López', Marie Angel Jimenez-Herrera², Roberto Grand³, Betty Bracho-Bracho4

\begin{abstract}
RESUMEN
Introducción: Las quemaduras constituyen una de las agresiones más graves contra el organismo y son uno de los problemas de salud más significantes a nivel mundial; Venezuela no escapa de esta situación en el 2011, se presentaron 39.955 casos de quemaduras que representan $0.2 \%$ de los pacientes atendidos en ese año, siendo esta misma estadística para el estado Falcón. La protocolización del tratamiento de las quemaduras establece en esta evaluación inicial la magnitud del daño, determinando la extensión y la profundidad de la quemadura y la evaluación secundaria. En el Hospital Universitario Dr. Alfredo Van Grieken no se encontró un protocolo de tratamiento para las quemaduras en el paciente adulto, aun cuando es un hospital Tipo IV. Objetivos: Diseñar un protocolo para el manejo inicial del paciente quemado en el Servicio de Cirugía General del Hospital Universitario Dr. Alfredo Van Grieken de Coro -Venezuela, noviembre - diciembre de 2015. Métodos: Proyecto factible en donde se evaluaron características operativas de H.U.A.V.G. así como diferentes protocolos de manejo del paciente quemado. Resultados: Se revisaron y analizaron los protocolos de manejo del quemado permitiendo definir fortalezas debilidades y adaptabilidad a las características operativas del H.U.A.V.G en el cual no es posible el manejo del usuario con quemaduras graves. Conclusión: El H.U.A.V.G. posee características operativas para el manejo inicial de las quemaduras en todas las categorías, sin embargo de estas las del grupo III y IV deben ser trasladadas a una unidad de atención especializada en quemados, basados en lo propuesto por nuestro protocolo.
\end{abstract}

Palabras clave: Quemaduras; Protocolos; Manejo Inicial. (fuente: DeCS BIREME).

\begin{abstract}
Introduction: Burns are one of the most serious attacks against the body and are one of the most significant problems of global health; Venezuela does not escape this situation in 2011, 39.955 burn cases representing 0.2\% of patients treated in that year were presented, with the same statistics for the state of Falcon. The formalization of the treatment of burns forth in this initial evaluation the extent of damage, determining the extent and depth of the burn and the secondary assessment. At the University Hospital Dr. Alfredo Van Grieken a treatment protocol for burns in adult patients was found, even though it is a type IV hospital. Objective: To design a protocol for the initial management of patients burned in the Department of General Surgery, University Hospital Dr. Alfredo Van Grieken Coro -Venezuela, November-December 2015. Methods: Project where feasible operational characteristics were evaluated HUAVG different protocols and burned patient management. Results: There were reviewed and analyzed burned management protocols define strengths and weaknesses allowing adaptability to the operating characteristics of HUAVG in which it is not possible to operate the user with severe burns. Conclusions: HUAVG It has operating for initial management of burns in all categories features, however these the group III and IV should be transferred to a unit specializing in burn care, based on our proposed protocol.
\end{abstract}

Key words: Burns; Protocols; Preliminary treatment. (source: MeSH NLM).

\footnotetext{
'Especialista en Cirugía General Hospital Universitario “Dr. Alfredo Van Grieken" Coro estado Falcón- Venezuela.

${ }^{2}$ Especialista en Anestesiología Hospital Universitario de Caracas Distrito Capital - Venezuela.

${ }^{3}$ Especialista en Cirugia Plastica y Reconstuctiva Hospital Universitario “Dr. Alfredo Van Grieken” Coro estado Falcón- Venezuela.

${ }^{4}$ Doctor en metodología de la investigación Hospital Universitario “Dr. Alfredo Van Grieken"Coro estado Falcón- Venezuela
}

Correspondencia: Germánico Fuentes López. Dirección: Universidad Nacional Experimental "Francisco de Miranda", Coro Estado Falcón. Correo:germanicofl@gmail.com

Citar como: Germánico Fuentes-López, Marie Angel Jimenez-Herrera, Roberto Grand, Betty Bracho-Bracho. Protocolo para el manejo inicial del paciente quemado hospital universitario Dr. Alfredo Van Grieken Coro-Venezuela, Noviembre-Diciembre 2015 [Artículo Original]. Rev. Fac. Med. Hum. $2016 ; 16(3): 38-45$. DOI 10.25176/RFMH.v16.n3.651 


\section{INTRODUCCIÓN}

Las quemaduras constituyen una de las agresiones más graves contra el organismo, ya que, dependiendo de la cantidad de tejidos destruidos o de su localización en estructuras vitales, pueden llevar al paciente a la muerte, y su morbimortalidad está directamente relacionada con la calidad de la terapia aplicada. De hecho, se estima que de cada 100.000 habitantes, alrededor de 300 personas sufren cada año quemaduras que requieren atención sanitaria, y cerca del $5 \%$ precisan cuidados hospitalarios ${ }^{1-8}$.

Así, las quemaduras son lesiones traumáticas que conducen a necrosis hística de variable extensión y profundidad; que son causadas por agentes que pueden ser de naturaleza física, química o biológica y que provocan alteraciones hístico-humorales capaces de desencadenar en la muerte o dejar secuelas invalidantes o deformantes en el paciente que la sufre, derivando además en síntomas emocionales de diversa intensidad, y ocasionando altos costos económicos, emocionales y familiares, por requerir medicamentos, y procedimientos múltiples, además de estancia hospitalaria prolongada ${ }^{9-14}$.

Según datos de la Organización Mundial de la Salud en 2002 hubo 320000 muertes por quemaduras en el mundo y para 2007 más de 500.000 lo que demuestra el incremento en este tipo de lesión. Del mismo modo, la Asociación Americana de Quemaduras reportó que entre los años 2000 y 2010 hubo 148.000 egresos hospitalarios por quemaduras, 5.540 muertes y un gasto aproximado por tratamiento de pacientes quemados de 1.000 millones de dólares al año 25,26 .

En Venezuela, son escasas las publicaciones actualizadas sobre quemaduras que permiten definir estadísticas significativas. Según el Anuario de Morbilidad de 2011, ese año ocurrieron 39.955 casos de quemaduras, lo que representó el 0,2\% de todas las consultas a nivel nacional, en el Estado Falcón para ese mismo año se reportaron 1.853 casos constituyéndose en el $0,2 \%$ de la morbilidad ${ }^{27-29}$.

En el Hospital Universitario Dr. Alfredo Van Grieken del Estado Falcón en 2014 se atendieron 474 pacientes politraumatizados, de los cuales $16,66 \%$ (79/474) presentaron algún tipo de quemadura, de los cuales el $23 \%$ fue atendido en el Servicio de Emergencias de Adultos y $77 \%$ en el Servicio de Emergencia Pediátrica ${ }^{30}$.

En Venezuela es muy poco lo que se ha avanzado al referirse a un plan de desarrollo para la atención del paciente quemado. De hecho, en la actualidad se cuenta con pocos centros asistenciales que cuentan con una Unidad de Quemados como tal. Entre estos están: Hospital Coromóto en el Estado Zulia que posee Unidad de Quemados, el Hospital Dr. Domingo Luciani en Caracas Distrito Capital, entre otros pocos centros, que cuentan con disponibilidad de terapia intensiva para la atención del paciente quemado y camas para quemados con quemaduras menores, y en el resto del país; la mayoría de los hospitales carecen de servicios especiales de quemados, como es el caso del Hospital Universitario Dr. Alfredo Van Grieken ${ }^{14}$.

Las quemaduras pueden clasificarse considerando la profundidad y la extensión, Usando para esto diferentes métodos de estimación como son la regla de Wallace (Regla de los 9), el esquema de Lund-Browder, la regla de la palma de la mano ( la palma representa $1 \%$ de SCT) y los grupos de gravedad propuestos en la clasificación del Dr. Fortunato Benaim, por mencionar algunos $^{3,7,15,24,26,31}$.

En la valoración primaria del paciente quemado comienza en primera instancia tras el trauma térmico, y está basada en el principio de que el paciente gran quemado debe tratarse como paciente politraumatizado, y comienza siguiendo la secuencia $A B C$, prestando atención a la vía aérea $(A)$, la respiración (B) y la circulación (C) $)^{3,24}$.

Postergándose la evaluación de la gravedad de las quemaduras, para cuando el paciente se encuentre estable. Siendo fundamental valorar e incluir junto con el estado general y actual de salud unos datos específicos como son: hora cero u hora aproximada en la que se produjo la lesión, agente de la lesión, tiempo de contacto, recinto cerrado o abierto, y mecanismo del accidente. Determinándose además, la extensión, la profundidad y la localización ${ }^{3,24}$.

La protocolización del tratamiento de las quemaduras establece en esta evaluación inicial la magnitud del daño, determinando la extensión y la profundidad de la quemadura y en la evaluación secundaria, una vez estabilizado el paciente y colocado en un sitio seguro, debeevaluarse deforma más detalladatodoslosórganos y sistemas, descartándose lesiones o traumatismos asociados que ameriten tratamiento inmediato, como la estabilización cervical o corporal, considerándose la extensión, profundidad, y tratamiento de quemaduras de primer, segundo y tercer grado, eléctricas y químicas en el sitio del accidente, en la sala de emergencia ${ }^{33-35}$.

En el manejo inicial del paciente con quemaduras se ha determinado que entre las primeras medidas que se deben tomar en cuenta son: 1) Reanimación (ABC): 
vigilar las constantes vitales, ventilación y perfusión, 2) Analgesia: AINES, opiáceos, 3) Protección gástrica y sonda nasogástrica y vesical si la SCQ es > 25\%,4) Hidratación: según la fórmula de Parckland (SCQ x 4 x peso en kg: $\mathrm{ml}$ de Ringer lactato en 24 horas), 4) Profilaxis antitetánica si procede, 5) Elevación de la región quemada y enfriamiento con suero salino para detener la progresión de la quemadura, 6) Curas locales, 7) No profilaxis antibiótica de entrada, salvo en quemaduras eléctricas, 8) Resto de tratamiento dependerá de la gravedad de las quemaduras ${ }^{24,31}$.

Sin embargo, a pesar de los avances tecnológicos en las diferentes áreas de la medicina que han favorecido un aumento en la sobrevida de los pacientes con quemaduras severas, todavía se continúan reportando estancias hospitalarias prolongadas y alto porcentaje de complicaciones y mortalidad ${ }^{6,37,38}$.

Por lo que, se han diseñado diferentes protocolos de atención al paciente afectado con quemaduras, entre los que se encuentran los siguientes: Protocolo para el Manejo del Paciente Quemado Crítico en el Área Prehospitalaria propuesto en la Universidad CES de Medellín, el del Servicio Andaluz de Salud, protocolo de atención al paciente quemado del Hospital Universitario Regional y el del Hospital Verge; el presentado por el Gobierno Federal de México y el del Ministerio de Salud de Chile $\mathrm{C}^{40-45}$.

El Hospital Coromoto de Maracaibo - Venezuela ha publicado el Protocolo de Actuación ante Pacientes con Quemaduras donde se describe el tratamiento de las quemaduras superficiales $A$ (I grado), quemaduras AB (II grado), quemaduras B profunda (III grado), manejo de excisión y cobertura, manejo de la zona dadora, manejo de posible lesión inhalatoria, y tratamiento ambulatorio ${ }^{46}$.

En el Hospital Universitario Dr. Alfredo Van Grieken no se encontró un protocolo de tratamiento para las quemaduras en el paciente adulto, aun cuando es un hospital Tipo IV formador de especialistas en cirugía general con adiestramiento en el manejo del paciente con quemaduras, que cuenta con Unidad de Cuidados Especiales, Unidad de Cuidados Intensivos, 79 camas en el Departamento de Cirugía, y recurso humano especializado, por lo que resulta pertinente y viable el diseño y la estandarización del protocolo del manejo inicial del paciente quemado para engranar los factores que garanticen, un adecuado diagnóstico para un efectivo tratamiento de esta población ${ }^{30}$.

Luego de una revisión exhaustiva en donde se hayan realizado protocolos de atención al paciente quemado, no se encontró ninguna en el cual se explique el fundamento del diseño de los mismos. Sin embargo, se presenta este estudio que fue realizado en Venezuela y que muestra algunas características del paciente quemado, como es: Urdaneta y col. (2009) en Venezuela realizaron un estudio descriptivo, retrospectivo con para determinar las características epidemiológicas y etiológica del paciente quemado, por lo que revisaron 120 historias clínicas de pacientes con quemadura atendidos en la División de Cirugía Plástica, Reconstructiva y Maxilofacial del Servicio Autónomo Hospital Universitario de Maracaibo, evaluando variables como: edad, sexo, etiología, tasas de morbimortalidad, severidad, porcentaje comprometido, y tiempo de estancia hospitalaria, reportando que el $71,7 \%$ fueron hombres, grupo de edad más afectado 20 a 29 años, el accidente ocurrió mas frecuentemente en el hogar 45\%, 51,7\% sufrieron quemaduras por fuego directo, 37\% presentaron \%SCQ entre 15 - 20, estancia hospitalaria superior a 31 a 60 días fue del $33 \%{ }^{15}$.

Por su parte, Sánchez y col. (2015) en Venezuela describieron el manejo de quemados en masa en situación del desastre ocurrido en el Complejo Refinador de Paraguaná en el 2012, en el que incluyeron el total de los 229 pacientes afectados y tratados en el Hospital Universitario Dr. Rafael Calles Sierra del IVSS de Punto Fijo que sufrieron lesiones térmicas, por inhalación y por onda expansiva, expresando que en el lugar del desastre se estableció un escenario de Triage Pre-Hospitalario donde el personal paramédico entrenado, categorizó a las víctimas de acuerdo a la extensión y profundidad de las quemaduras, la severidad de las lesiones asociadas y lesiones por inhalación; considerando la distancia entre el lugar de los hechos y los diferentes centros de salud adyacentes (Nivel I, II y III de Atención Hospitalaria) y establecieron prioridades de evacuación ${ }^{47}$.

Las quemaduras son un problema de salud de alto costo, en vista de que representa una forma devastadora del traumatismo. La existencia de protocolos internacionales para el manejo multidisciplinario del paciente y la existencia de mejores, y más unidades especializadas ha representado un incremento fundamental en la sobrevida de los usuarios con dicha noxa ${ }^{1-47}$.

En Venezuela se cuentan con pocas unidades especializadas en el manejo de quemados, siendo el Hospital Coromoto, específicamente el Centro de Atención Integral del Paciente Quemado (CAINPAQ) centro de referencia nacional, ubicado en el estado 
Zulia, el más cercano a nuestro nosocomio; y teniendo en cuenta que: el Hospital Universitario Dr. Alfredo Van Grieken que es un hospital de IV nivel y de referencia de la región, en la Península de Paraguaná se ubica específicamente en Punto Fijo estado Falcón, la Refinería de Amuay sitio de asentamiento ocasional de accidentes laborales que involucra las quemaduras, y que en el Hospital Universitario Dr. Alfredo Van Grieken no cuenta con un protocolo estandarizado para el manejo inicial del paciente quemado; resulta imperioso el establecimiento de este para la optimización del manejo de dichos pacientes de forma multidisciplinaria ${ }^{30,46}$.

El manejo multidisciplinario y protocolizado del paciente quemado influye de manera directa en la disminución de la tasa de morbimortalidad de los pacientes que sufren estas afecciones, destacando tres aspectos fundamentales para el tratamiento oportuno y efectivo de los pacientes como son: soporte físico (planta física, sistemas de circulación, equipamiento e insumos), recursos humanos (dotación, capacitación) y organización (estructura organizativa, procesos, protocolo terapéutico). En el Hospital Universitario Dr. Alfredo Van Grieken de Coro - Venezuela como centro asistencial de IV nivel, se cuenta con el Departamento de Cirugía, en el cual existen 79 camas para hospitalización además de Unidad de Cuidados Especiales y Unidad de Cuidados Intensivos para el manejo de pacientes en condiciones de cuidado, con insumos médico-quirúrgicos y que con el advenimiento del convenio interinstitucional entre el Hospital Coromoto de Maracaibo y el Hospital Universitario de Coro Dr. Alfredo Van Grieken, en donde se adiestra al cursante del postgrado de cirugía general en la Unidad de Caumatología en el manejo del paciente quemado, que junto a la existencia de un cirujano plástico experimentado adjunto al departamento de cirugía, resulta pertinente y viable la estandarización del protocolo de manejo inicial del paciente quemado, permite engranar los factores que garanticen, un adecuado diagnóstico y tratamiento efectivo de este grupo poblacional.

Para esto nuestro objetivo fue, diseñar un protocolo para el manejo inicial del paciente quemado en el Servicio de Cirugía General del Hospital Universitario Dr. Alfredo Van Grieken de Coro-Venezuela, noviembre 2015 - enero 2016.

\section{MÉTODOS}

Se trata de un Proyecto factible, basados en una investigación de tipo documental, con un diseño no experimental.

Poblacióny Muestra:Portratarse deun proyectofactible fundamentado en una investigación documental, en este estudio se evaluaron documentos contentivos de las características operativas del Hospital Universitario Dr. Alfredo Van Grieken y se revisaron diferentes protocolos diseñados, desarrollados y ejecutados en diferentes centros asistenciales internacionales y nacionales que permitieron diseñar un protocolo de atención para el paciente quemado en el Hospital Universitario Dr. Alfredo Van Grieken de Coro. Técnica de Recolección de Datos: Por tratarse de un proyecto factible se desarrolló en dos (2) etapas, luego de que el Proyecto del Trabajo de Investigación fue evaluado y aprobado por el Comité de Bioética para la Investigación en Seres Humanos de la UNEFM y por la Comisión de Revisión de Proyectos del Trabajo Especial de Grado del Programa de Postgrado de Cirugía General se aplicó un instrumento denominado Formulario de Recolección de Datos (Anexo 1) que consta de dos partes. En la parte I, se anotaron las características operativas del Hospital Universitario Dr. Alfredo Van Grieken que deben ser consideradas en el tratamiento inicial del paciente adulto quemado, tales como soporte físico (planta física, sistema de circulación, equipamiento e insumos), recursos humanos (dotación, capacitación) y organización (estructura organizativa, procesos, protocolos. En la parte II, se analizaron de diferentes protocolos de atención al paciente quemado anotándose sus fortalezas, debilidad y adaptabilidad al Hospital Universitario Dr. Alfredo Van Grieken. El beneficio de este estudio radica en que los datos obtenidos permitieron diseñar un protocolo de atención al paciente quemado en un centro asistencial de referencia regional y formador de especialistas en cirugía general, lo que logrará la estandarización en el tratamiento de esta patología que presenta altas tasas de morbimortalidad.

\section{Técnica de Análisis de Datos}

Los datos obtenidos se analizaron para diseñar un protocolo para el manejo inicial del paciente quemado en el Servicio de Cirugía General del Hospital Universitario Dr. Alfredo Van Grieken de Coro - Venezuela. 


\section{RESULTADOS}

Se realizó una investigación documental mediante entrevista estructurada a las autoridades del Hospital Universitario Dr. Alfredo Van Grieken para establecer las características operativas de este centro asistencial de referencia regional para tratar al paciente adulto considerando planta física, recursos humanos y organización (estructura organizativa, procesos, protocolo), estableciéndose en cuanto a la Planta Física: Servicio de Emergencia de Adultos, 1 Consultorio para Cirugía General en el Área de Emergencia, Pabellones de Emergencia, Sala de Shock y Unidades de Cuidados Especiales, Salas de Observación en Emergencia de Adultos, Sala de Aislamiento, Sala de yeso, Servicio de radiología, Laboratorio de bioanálisis, Área Quirúrgica, - Unidad de Cuidados Intensivos, Banco de Sangre, Servicio de Cirugía, Tabla 1.

Los recursos humanos del Hospital Universitario Dr. Alfredo Van Grieken establecidos fueron: Especialista en Cirugía General, Especialista en Cirugía Plástica,
Residentes de Postgrado en Cirugía General UNEFM, Licenciado en Nutrición y Dietética, Especialistas en Medicina Crítica, Especialistas en Anestesiología, Especialistas en Medicina Física y Rehabilitación, Fisioterapeutas, Foniatra, Especialistas en Psiquiatría, Especialistas en Nefrología, Especialistas en Cardiología, Especialistas en Radiología, Especialistas en Neumonología, Enfermeras especializadas en Medicina Crítica, Hemoterapia, Cirugía y Técnicos radiólogos, Tabla 1. La organización del Hospital Universitario Dr. Alfredo Van Grieken es de tipo vertical, Tabla 1. 27 En cuanto a la presencia de Procesos/ Protocolos para paciente quemados se determinó que no existen en el Hospital Universitario Dr. Alfredo Van Grieken, Tabla 1.Se revisaron y analizaron 15 protocolos de manejo y atención del paciente quemado ${ }^{1,12,14,19,22,}$ $24,33,34,36,40,43-46$ y esto permitió establecer sus fortalezas, debilidades y determinar la adaptabilidad de los mismos a la realidad medico asistencial del Hospital Universitario Dr. Alfredo Van Grieken, Tabla 2.

Tabla 1. Características operativas para tratar al paciente quemado, Servicio de Cirugía General, Hospital Universitario Dr. Alfredo Van Grieken Coro -Venezuela, noviembre 2015 - enero 2016.

\section{Planta física}

- Servicio de Emergencia de Adultos

- 1 Consultorio para Cirugía General en Área de Emergencia (óptimas condiciones)

- 2 Pabellones de Emergencia en Área de Emergencia (condiciones óptimas)

- Área de Hospitalización en Área de Emergencia

- Sala de Shock y Unidades de Cuidados Especiales.

- Salas de Observación para mujeres y hombres en Área de Emergencia

- Sala de Aislamiento en Área de Emergencia
- Sala de yeso en Área de Emergencia

- Servicio de radiología: 3 equipos de radiología (1 operativo), Tomógrafo (no operativo), 1 sala de ecografía

- Laboratorio de bioanálisis (óptimas condiciones)

- Área Quirúrgica: 5 quirófanos (4 operativos), 1 área de recuperación post anestésica (operativa)

- Unidad de Cuidados Intensivos: 6 camas (5 operativas), sin ambiente de aislamiento

- Banco de Sangre (operativo)

- Servicio de Cirugía: 79 camas operativas

- 6 Ascensores: 2 operativos

\section{Recursos humanos}

- Especialista en Cirugía General: 10

- Especialista en Cirugía Plástica: 1

- Residentes de Postgrado en Cirugía General UNEFM: 29

- Licenciado en Nutrición y Dietética: 1

- Especialistas en Medicina Crítica: 11

- Especialistas en Anestesiología: 15

- Especialistas en Medicina Física y Rehabilitación: 4

- Fisioterapeutas: 2
- Foniatra: 1

- Especialistas en Psiquiatría: 3

- Especialistas en Nefrología: 6

- Especialistas en Cardiología: 9

- Especialistas en Radiología: 3

- Especialistas en Neumonología: 6

- Enfermeras especializadas en Medicina Crítica, Hemoterapia, Cirugía

- Técnicos radiólogosi

Organización:

Vertical

Procesos/Protocolos para paciente quemados:

No existen

Fuente: Autoridades Hospital Universitario Dr. Alfredo Van Grieken 
Tabla 2. Análisis de protocolos para el manejo del paciente quemado. Servicio de Cirugía General, Hospital Universitario Dr. Alfredo Van Grieken Coro -Venezuela, noviembre 2015 - enero 2016

\section{Protocolos de manejo y atención del paciente quemado}

- Clasifican las quemaduras consi- • No se apreciaron debilidades derando profundidad, extensión y importantes en su aplicabilidad. gravedad.

- Establecen la valoración, tratamiento y manejo de las quemaduras considerando el tipo (leve, moderada y grave)

- Definen los criterios de hospitalización y/o traslado a unidades de quemados
- Los protocolos se presentan de manera que pueden ser adaptados en diferentes centros asistenciales considerando su capacidad resolutiva.

Fuente: Protocolos de manejo y atención del paciente quemado 1, 12, 14, 19, 22, 24, 33, 34, 36, 40, 43 - 46

\section{DISCUSIÓN}

Las lesiones graves por quemaduras representan la injuria más devastadora que puede sufrir un paciente, por ser lesiones producidas en los tejidos vivos por la acción de diversos agentes físicos que provocan alteraciones que van desde un simple eritema hasta la destrucción total de las estructuras ${ }^{45}$.

Para el manejo del paciente quemado es necesario contar con un centro asistencial que presente ciertas características operativas, como una cama para pacientes quemados por cada 200.000 habitantes, atención continua cubierta 24 horas al día, 365 días al año, al menos por un especialista en Cirugía Plástica, Estética y Reconstructiva de presencia física en el hospital entre otros. Sin embargo, luego del análisis de los resultados obtenidos durante esta investigación se determinó que en el Hospital Universitario Dr. Alfredo Van Grieken pese a ser el centro de referencia del estado Falcón, y que en esta región encuentra en funcionamiento una de las refinerías de petróleo más grandes del mundo, como es el Complejo Refinador Paraguana, ubicado en la ciudad de Punto Fijo; no cuenta con los medios para la atención sistemática y protocolizada del paciente quemado, de tal manera, que la morbimortalidad de los pacientes con grandes quemaduras puede aumentar, así como las secuelas derivadas de estas lesiones ocasionando un incremento del gasto del sector salud para el tratamiento de dichas repercusiones.

Del mismo modo, los centros especializados en atención del paciente quemado se encuentran en las grandes ciudades del país, como es el caso del Hospital
Coromoto ubicado en la ciudad de Maracaibo en el estado Zulia, y estos no son suficientes para brindar atención a la alta demanda de 31 quemados en el país, por lo que con frecuencia se ha visto que en Hospitales del Interior del país no hay cirujanos plásticos ni caumatológos, y debido a la ausencia de esta figura, los cirujanos generales, sin entrenamiento formal en el manejo de pacientes quemados se ven involucrados.

Por estas razones, el tratamiento definitivo se retrasa, ya que los pacientes deben viajar largas distancias en medios de transporte deficientes, para recibir los cuidados de un equipo multidisciplinario de especialistas para pacientes quemados ${ }^{47}$.

Por tal motivo es imperativo el establecimiento $y$ puesta en funcionamiento de procesos $\mathrm{y} / \mathrm{o}$ protocolos adaptados a la realidad del Hospital Universitario Dr. Alfredo Van Grieken que permitan estandarizar el manejo inicial del paciente quemado y así brindar bienestar biopsicosocial a los que sufren las injurias térmicas.

\section{CONCLUSIÓN}

las características operativas del Hospital Universitario Dr. AlfredoVan Grieken para tratar al paciente quemado establecen que se puede asistir en la fase inicial al usuario que sufre esta noxa ya que posee personal entrenado en esta patología, y servicios auxiliares e insumos para brindar el soporte al tratamiento inicial. El análisis de diferentes protocolos para el manejo del paciente quemado determinó que estos definen las quemaduras según su profundidad, su extensión, el agente causal, criterios de ingreso hospitalario y en 
unidades de atención especializada, estandarizando el manejo durante la estancia en el área de emergencia que involucra desde el triage hasta el tratamiento de estos pacientes. El protocolo para el manejo inicial del paciente quemado en el Hospital Universitario Dr. Alfredo Van Grieken diseñado que incluye evaluación primaria, evaluación secundaria, clasificación de las quemaduras, criterios de hospitalización, tratamiento y manejo de las quemaduras leves, moderadas y graves y que se presenta como un anexo.
Financiamiento: Autofinanciado.

Conflicto de interés: Los autores declaran no tener conflictos de interés en la publicación de este artículo.

Recibido: 04 de noviembre

Aprobado: 26 de diciembre

\section{REFERENCIAS BIBLIOGRÁFICAS}

1. Guerra J. Enfoque inicial del paciente quemado. Revista Científica Salud Uninorte 2013; 8: 0-0.

2. Velasco García D, Méndez Torres F. Características clínicas y epidemiológicas de las quemaduras en pacientes ingresados en la unidad de quemados. Hospital Luis Vernaza, Guayaquil. 2009-2010. Repositorio Digital de la Universidad de Cuenca-2013. [Septiembre, 2015] Disponible en: http://dspace.ucuenca.edu.ec/handle/123456789/20053

3. Villanueva Torres B. Asistencia de enfermería de los pacientes con quemaduras. Repositorio Institucional de la Universidad de Málaga-2014. [Septiembre, 2015] Disponible en: http://dspace.uma.es/xmlui/handle/10630/7733

4. Solís F, Domic C, Saavedra R, González MA. Incidencia y prevalencia de las lesiones por quemaduras en menores de 20 años. Rev. chil. pediatr. 2014; 85 (6): 674-681.

5. Sciaraffia C, Andrades P, Pamela W. Quemaduras. [Septiembre, 2015] Disponible en: https://www.redclinica.cl/Portals/0/Users/014/14/14/Publicaciones/quemaduras.pdf

6. Ortiz Prado E, Rubio Gallegos F, Rodríguez E. Análisis epidemiológico de quemaduras en el paciente adulto ingresado en la Unidad de Quemados del Hospital Eugenio Espejo, Quito Ecuador, durante el periodo 2005-2011. [Septiembre, 2015] Disponible en: http://www.researchgate. net/profile/Esteban_Ortiz-Prado/publication/270273417_ANALISIS_EPIDEMIOLOGICO_DE_QUEMADURAS_EN_EL_PACIENTE_ADULTO_INGRESADO_EN_LA_UNIDAD_DE_QUEMADOS_DEL_HOSPITAL_EUGENIO_ESPEJO_QUITO_ECUADOR_DURANTE_EL_PERIODO_2005-2011/ links/54a414960cf256bf8bb31b18.pdf

7. Gómez Nava KA. Valoración preanestestésica del paciente gran quemado. Rev Mex Anest 2014; 37 (S1): 212-214

8. Salvador Sanz J.F., Novo Torres A., Lorda Barraguer E., Castillo F., Torra i Bou J.E., Torregrosa Ramos M.J. Estudio comparativo de efectividad de un apósito de plata nanocristalina frente a sulfadiazina argéntica en el tratamiento de pacientes quemados. Cir. plást. iberolatinoam. 2011; 37 (3): 253-266.

9. Collado Hernández CM, Pérez Núñez V, Lorente Gil SR, Pérez Suárez FA. Caracterización clínica epidemiológica de las quemaduras del adulto mayor ingresado en el Hospital Celia Sánchez. CCM 2015; 19 (3): 396-405.

10. Fernández Vega FL, Puebla Farigola I, Carrillo Vázquez L. Alteraciones psicológicas en pacientes ingresados por quemadura. Multimed. Revista Médica. Granma 2015; 19 (1): 0-0.
11. Álvares Millán S, García Collado F, Ramírez Pizano AM, Rivera Fernández C, García Murillo M, Franco García EM. Quemaduras dérmicas superficiales: pauta de actuación con apósito primario único de hidrofibra Ag en Atención Primaria. Enferm Dermatol. 2014; 8 (22): 10-21.

12. Berrocal Revueltas $M$, Guette Viiana AM, Rodríguez Romero P, Rodríguez Torres GM, Ruiz Pérez MÁ, Salgado Monterrosa EJ. Paciente quemado: Manejo de urgencias y reanimación. Rev. cienc. biomed. 2011; 2 (2): 316-326.

13. Camacho Valdez GA. Gutiérrez Beltrán MR, Estrada A, Martínez Tapia ME. Estabilidad hemodinámica transoperatoria en pacientes con quemadura extensa. Con uso de sufentanil comparado con fentanil. Universidad Autónoma de Chihuahua-2012. [Septiembre, 2015] Disponible en: http:// www.fm.uach.mx/posgrado/2012/01/18/Tesis\%20Dra.\%20Camacho.pdf

14. Zapata Sirvent RL, Jiménez Castillo CJ, Besso J, editores. Quemaduras. Tratamiento crítico y quirúrgico. Actualización 2005. Caracas: Editorial Ateproca 2005; 1-6.

15. Urdaneta Lima E, González de Boscán TA. Características epidemiológicas y etiológicas de las Quemaduras en Pacientes Adultos. Trabajo Especial de Grado para optar al Grado Académico de Especialista en Cirugía Plástica y reconstructiva LUZ - 2011. [Septiembre, 2015] Disponible en: http://tesis.luz.edu.ve/tde_arquivos/16/TDE-2011-1101T09:06:53Z-2068/Publico/urdaneta_lima_eleazar.pdf

16. Espinoza Machado BM, Castillo Calderón GJ, Padrón Cordero LM, González AN, Borroto Guevara M. Intervención educativa sobre quemaduras por agentes físicos al personal de enfermería del Policlínica Universitario Norte de Morón. MEDICIEGO 2013; 19 (S1): 0-0.

17. Navarro Medina JF. Uso de heparina sistémica y tópica en el tratamiento de quemaduras en el Hospital General de Querétaro. Repositorio Institucional Universidad Autónoma de Querétaro - 2015. [Septiembre, 2015] Disponible en: http://ri.uaq.mx/handle/123456789/2613

18. Carrión Dávila T, Cevallos Pinzón MC. Etiología de las quemaduras y perfil microbiológico encontrado en las muestras de pacientes de la Unidad de Quemados del Hospital Regional Isidro Ayora, período abril - septiembre 2012. Repositorio Universidad Nacional de Loja - 2012. [Septiembre, 2015] Disponible en: http://dspace.unl.edu.ec/jspui/handle/123456789/7857

19. Castañeda Quintero PA, Marín Torres Mc, Morantes Pérez LF, Ospina Mejía JL, Velasquez Delgado A, Toro Palacio LF. Protocolo del paciente traumatizado por quemadura térmica en el ámbito prehospitalario. Repositorio Digital Universidad CES-2012. [Septiembre, 2015] Disponible en: http://bdigital.ces.edu.co:8080/repositorio/handle/10946/262 
20. Schencke C, Salvo J, Vasconcellos A, Del Sol M. Estudio Comparativo de la Cicatrización en Quemaduras con Tratamiento en Base a Miel de Ulmo (Eucryphia cordifolia) y Vitamina C oral versus Hidrogel en Cobayos (Cavia porcellus). Int. J. Morphol. 2013; 31 (3): 839-844.

21. Camuci MB, Trevisan Martins J, Narciel Cardeli AA, Cruz Robazzi ML. Caracterización epidemiológica de pacientes adultos internados en Unidad de Terapia Intensiva de Quemados. Cogitare Enferm. 2014; 19 (1): 78-83.

22. Píriz Campos RM, Martín Espinosa NM, Postigo Mota S. Guía terapéutica en el paciente quemado crítico. Rev Enferm 2014; 37 (2): 111-114.

23. Vidaurreta Lara N, Suarez Morales O, García Urquijo A, Peláez Quintana G. Caracterización clínico-epidemiológica y microbiológica del paciente quemado leve con infección local. Acta Médica del Centro 2014; 8 (4): 0-0.

24. Gorordo Del Sol LA, Hernández López GD, Zamora Gómez SE, García Román MT, Jiménez Ruiz A, Tercero Guevara Bl. Atención inicial del paciente quemado en UCI: revisión y algoritmo. Rev Hosp Jua Mex 2015; $82(1): 43-48$

25. Albornoz CR, Villegas J, Peña V, Whittle S. Epidemiología del paciente gran quemado adulto en Chile: experiencia del Servicio de Quemados del Hospital de la Asistencia Pública de Santiago. Rev. méd. Chile 2013 141 (2): 181-186.

26. Montoya T. Hipnosis para el control del dolor en pacientes con quemaduras. Ajayu 2010; 8 (2): 0-0.

27. Álvarez M, Silva J. Quemados en Uruguay, Perfil Epidemiológico en Uruguay. Archivos Catarinenses de Medicina 2009; 38 (1): 55-57.

28. Moya Rosa EJ, Faces Sánchez M. Lesión por quemadura en el adulto mayor. Arch. méd. Camaguey 2011; 15 (5): 0-0.

29. Ministerio del Poder Popular para la Salud - Venezuela. Anuario de Morbilidad 2011. [Septiembre, 2015] Disponible en: http://www.bvs.gob. ve/anuarios_de_morbilidad/ANUARIOSDEMORBILIDAD2011.pdf

30. Hospital Universitario Dr. Alfredo Van Grieken. Estadística Vital. No publicado.

31. Moraleda E, García Pumarino R, Marín M, Sánchez Olaso A. Tratamiento de las quemaduras. En: Manual de Urgencias Quirúrgicas. [Septiembre, 2015] Disponible en: http://irycis.org/doc/Publicaciones/Manual_Urgencias_Quirurgicas_4Ed.pdf\#page $=407$

32. Mangelsdorff G, García Huidobro MÁ, Nachari I, Atenas O, Whittle S, Villegas J. La quemadura eléctrica por alto voltaje es un factor predictor de mortalidad en pacientes "grandes quemados". Rev. méd. Chile 2011; 139 (2): 177-181.

33. Hospital General de Valencia. Protocolo de Tratamiento de Quemaduras en Atención Primaria. [Enero, 2016] Disponible en: http://chguv. san.gva.es/documents/10184/28508 Protocolo+de+tratamiento+de+quemaduras+en+atenci\%C3\%B3n+primaria/e3aa4e46-c64f-4c5d-8eee62b1b691aae7

34. Zapata Sirvent RL. Pautas de Tratamiento del Paciente Quemado. En: Congreso de Red de Sociedades Científicas - RSCMV VI Encuentro 2004. [Enero, 2016] Disponible en: https://www.researchgate.net/publication $/ 237347980$
35. Zapata Sirvent RL. Atención pre hospitalaria del quemado. Manejo inicial, tratamiento y traslado. En: Quemaduras. Tratamiento crítico y quirúrgico. Actualización 2005. Caracas: Editorial Ateproca 2005; 19-26.

36. Lobo O, Guzmán J, Gil W, Duque I. Quemaduras, Manejo Inicial en el Instituto Autónomo Hospital Universitario de Los Andes I.A.H.U.L.A. Revista Digital de Postgrado, 2012; 1(2): 61-70.

37. Cuenca Pardo J, Álvarez Díaz CJ. Evaluación del índice de severidad de las quemaduras (ABSI) en pacientes atendidos en la Unidad de Quemados del Hospital de Traumatología «Dr. Victorio de la Fuente Narváez» del IMSS. Cir Plast 2013; 23 (1): 5-13.

38. Moctezuma Paz LE, Páez Franco I, Jiménez González S, Miguel Jaimes KD, Foncerrada Ortega G, Sánchez Flores AY, González Contreras NA, Albores de la Riva NX, Núñez Luna V. Epidemiología de las quemaduras en México. Rev Esp Méd Quir 2015; 20: 78-82.

39. Bedoya Zapata LM, Restrepo Correa AM, Rendón Cano YA. Protocolo para el Manejo del Paciente Quemado Crítico en el Área Prehospitalaria. [Septiembre, 2015] Disponible en: http://bdigital.ces.edu.co:8080/dspace/bitstream/123456789/153/1/protocolo_para_el\%20_manejo_paciente_quemado_critico_area_prehospitalaria.pdf

40. Universidad Industrial de Santander. Protocolo Manejo de Heridas por Quemaduras - 2008. [Septiembre, 2015] Disponible en: https://www.uis. edu.co/intranet/calidad/documentos/bienestar_estudiantil/protocolos/ TBE.02.pdf

41. Servicio Andaluz de Salud. Guía de Práctica Clínica para el Cuidado de Personas que sufren Quemaduras - 2011. [Septiembre, 2015] Disponible en: http://www.guiasalud.es/GPC/GPC_485_Quemados_Junta_Andalucia_completa.pdf

42. Hospital Regional Universitario Carlos Haya. Guía de Actuación ante el Paciente Quemado - 2006. [Septiembre, 2015] Disponible en: http:// www.eps-salud.com.ar/Pdfs/Guia_Paciente_Quemado.pdf

43. Hospital Verge dels Lliris. Protocolo de Actuación ante Pacientes Quemados - 2008. [Septiembre, 2015] Disponible en: http://alcoy.san.gva. es/cas/hospital/urgencias/PROTOCOLOS\%20MEDICOS/PT-08\%20v1\%20 -\%20Protocolo\%20de\%20actuaci\%C3\%B3n\%20ante\%20pacientes\%20 quemados.pdf

44. Gobierno Federal de México. Guía de Práctica Clínica: Diagnóstico y Tratamiento del Paciente "Gran Quemado" - 2009. [Septiembre, 2015] Disponible en: http://www.cenetec.salud.gob.mx/descargas/gpc/CatalogoMaestro/040_GPC_GranQuemado/IMSS_040_08_EyR.pdf

45. Ministerio de Salud de Chile. Guía Clínica Gran Quemado. Minsal 2007. [Septiembre, 2015] Disponible en: http://web.minsal.cl/portal/url/ item/7222d6a3774f3535e04001011f01482e.pdf

46. Chacín Paz TA. Protocolo de Actuación ante Pacientes con Quemaduras. [Septiembre, 2015] Disponible en: http://es.slideshare.net/tuchapa/ protocolo-quemaduras

47. Sánchez R, Marín I, López M, Suárez B, Martínez B. Manejo de Quemados en Masa en Situación de Desastre. [Enero, 2016] Disponible en: https://prezi.com/ruyd_nn3olcl/manejo-de-quemados-en-masa-valencia/ 